\title{
AVALIAÇÃO DA VIABILIDADE DO PÓLEN COMO POSSÍVEL INDICATIVO DE TOLERÂNCIA A ALTAS TEMPERATURAS EM GENÓTIPOS DE TOMATEIRO
}

\author{
ANDREIA CRISTINA T. FERREIRA SILVA ${ }^{1}$, IZABEL CRISTINA LEITE ${ }^{2}$ E LEILA \\ TREVIZAN BRAZ ${ }^{3}$
}

Departamento de Biologia Aplicada à Agropecuária, FCAV/UNESP

\begin{abstract}
RESUMO - O objetivo foi avaliar o comportamento de cinco cultivares (Carmen, IPA-5, Príncipe gigante, Santa Clara e TSW-10) e uma linhagem de tomateiro (CL5915), quanto à tolerância a altas temperaturas, por meio da viabilidade do pólen. O Experimento I constou na coleta do pólen dos diferentes genótipos e germinação em lâminas contendo um meio com $10 \%$ de sacarose e $100 \mathrm{mg} . \mathrm{L}^{-1}$ de ácido bórico. As flores foram levadas à câmara de germinação regulada nas temperaturas $30^{\circ} \mathrm{C}, 35^{\circ} \mathrm{C}$, $40^{\circ} \mathrm{C}$ e $45^{\circ} \mathrm{C}$ por $1,2,3$ e 4 horas. Foram avaliados a porcentagem de germinação do pólen e o comprimento do tubo polínico. O Experimento II constou no plantio dos genótipos em casa-de-vegetação e avaliação de caracteres agronômicos. Pela observação dos resultados obtidos por meio da técnica de germinação de pólen in vitro e para a porcentagem de fixação de frutos no cultivo in vivo em casa-devegetação, constata-se que, ao considerar as maiores temperaturas de exposição, a linhagem CL5915 foi a que apresentou a maior porcentagem de germinação de pólen e comprimento do tubo polínico, assim como a cultivar Santa Clara, inicialmente considerada como padrão de sensibilidade a alta temperatura, foi uma das que apresentaram menor porcentagem de germinação do pólen, como também menor fixação de frutos. Por outro lado, diferenças quanto aos resultados entre as técnicas in vitro e in vivo foram verificadas. Esse fato pode ser em virtude de as condições de temperatura na casa-de-vegetação não serem exatamente as mesmas em que os genótipos foram submetidos no laboratório, como por exemplo, permanecer um longo período na mesma temperatura, sem oscilações.
\end{abstract}

TERMOS ADICIONAIS PARA INDEXAÇÃO: Germinação in vitro, tomate, Lycopersicon esculentum.

\section{VIABILTY EVALUATION OF POLLEN AS POSSIBLE INDICATOR OF HIGH TEMPERATURE TOLERANCE IN TOMATO GENOTYPES}

\footnotetext{
ABSTRACT - The objective of this research was to evaluate the behavior of five cultivars ( Carmem, IPA- 5, Principe gigante, Santa Clara TSW - 10) and tomato line (CL5915), in relation to high temperature

Recebido em 6/4/2000 - Aceito em 10/7/2000

1. Engenheiro Agrônomo, Aluna de Doutorado, FCAV - UNESP Campus de Jaboticabal, Via de Acesso Prof. Paulo Donato Castellane, s/n, Jaboticabal - SP, 14.884-900.

2. Professora Assistente Dra., Departamento de Biologia Aplicada, UNESP Campus de Jaboticabal, Isabelcl@fcav.unesp.br.

3. Professora Assistente Dra., Departamento de Produção Vegetal, UNESP Campus de Jaboticabal, leilatb@fcav.unesp.br.
} 
tolerance, using pollen viability. In the Experiment I the pollen was picked from different genotypes and germinated in glass slide containing Sacarose $10 \%$ and boric acid $100 \mathrm{mgL}^{-1}$. The flowers were transferred to germination chamber with temperature regulated to $30^{\circ} \mathrm{C}, 35^{\circ} \mathrm{C}, 40^{\circ} \mathrm{C}$ and $45^{\circ} \mathrm{C}$ during 1 , 2, 3 and 4 hours. The parameters evaluated were pollen germination and polinic tube length. In the Experiment II the genotypes were grown in greenhouse and some agronomic trairs were analyzed. Based in pollen germination in vitro technique and the percentage of fruit set during growth in greenhouse, it was verified that exposition to higher temperatures, the line CL 5915 showed the highest pollen germination percentage and polinic tube length, like Santa Clara, that was initially considered as sensitivity standard to high temperature, it was one that showed low pollen germination percentage, as well low fruit set. In another way, difference in results were observed between techniques in vivo and in vitro were not verified.

ADDITIONAL INDEX TERMS: Germination in vitro, tomato, Lycopersicum esculentum.

\section{INTRODUÇÃO}

O tomateiro (Lycopersicon esculentum Mill.) é uma planta originária da América do Sul, onde era encontrada principalmente ao longo dos Andes, na Colômbia e ao norte do Chile (Tigchelaar, 1986), sendo uma das hortaliças mais importantes por sua ampla adaptação, popularidade, e por se constituir em um produto de alto valor nutritivo. Sua importância está assegurada pela larga utilização do seu fruto, rico em vitamina $\mathrm{C}$, aminoácidos e ácidos orgânicos (Anderlini, 1982), integrando a dieta da população tanto na forma "in natura" como na forma industrializada. A área cultivada com essa hortaliça no Brasil, em 1999, foi de aproximadamente 65.589 ha, com produção em torno de 3,14 milhões de toneladas, sendo o Estado de São Paulo o maior produtor, seguido de Goiás e Minas Gerais (Agrianual, 2000). O tomateiro é uma planta que exige boas condições de temperatura, luminosidade e umidade relativa do ar para se desenvolver satisfatoriamente. Assim, as condições climáticas influenciam de forma significativa os processos fisiológicos da planta, tais como florescimento e frutificação.

O estresse térmico é o principal fator que limita a produtividade e adaptação das culturas, principalmente se as temperaturas extremas coincidem com os estádios críticos do desenvolvimento da planta. Atualmente, quer seja pelo inadequado manejo do ambiente em cultivo protegido, quer pela necessidade de incorporar novas áreas à produção de tomate, tem-se percebido a submissão da planta ao estresse térmico, com reflexos negativos na produtividade. A exposição a temperaturas acima de $34^{\circ} \mathrm{C}$ na época de germinação do grão de pólen provoca redução na porcentagem de germinação e na taxa de crescimento do tubo polínico (Stevens e Rudich, 1978), determinando um menor número de frutos por planta. Segundo Abdalla e Verkerk (1968), a causa da não-fixação dos frutos é a ausência de polens viáveis, pois, sob altas temperaturas, a quantidade de pólen é drasticamente reduzida. Iwahori (1965) observou que em condições de alta luminosidade, com temperatura em torno de $20^{\circ} \mathrm{C}$, a meiose ocorreu nove dias antes da antese, enquanto que as injúrias no pólen foram mais severas quando as plantas foram expostas a alta temperatura 5 dias antes da antese, quando os grãos de pólen estavam se desenvolvendo. De acordo com Sugiyama et al.,, (1966) a fase do florescimento mais sensível à alta temperatura $\left(40^{\circ} \mathrm{C}\right.$, por 3 a 4 horas) foi durante a meiose ( 8 a 9 dias antes da antese) e na fertilização ( 1 a 3 dias após a antese).

Nesse sentido, o desenvolvimento de métodos para determinar a viabilidade do pólen é útil em programas de melhoramento, permitindo realizar-se avaliação prévia de genótipos sob condições de estresse térmico. Este trabalho tem por objetivo avaliar genótipos de tomateiro quanto a tolerância a altas temperaturas por meio da viabilidade do pólen, e verificar se os testes in vitro 
são úteis na seleção de plantas de tomate para melhor performance em ambiente de elevada temperatura.

\section{MATERIAL E MÉTODOS}

O experimento I foi conduzido em casade-vegetação com controle de temperatura (25/22 ${ }^{\circ} \mathrm{C}$-dia/noite), do Departamento de Tecnologia, e o experimento II foi conduzido em casa-de-vegetação com temperatura alta (máxima de 35,2 a $40,3^{\circ} \mathrm{C} / \mathrm{dia}$ ), pertencente à área experimental do Setor de Olericultura e Plantas Aromático-Medicinais do Departamento de Produção Vegetal.

Foram utilizadas cinco cultivares ('Santa Clara', 'IPA-5', 'Carmen', 'Príncipe Gigante', 'TSW10') e uma linhagem (CL5915) de tomateiro, as quais, em conjunto, serão referidas como genótipos. Esses estão inseridos dentro de grupos de similaridade distintos, estabelecidos pelo método de TOCHER a partir das distâncias generalizadas de Mahalanobis (Braz, 1995), exceto 'IPA-5', sendo 'Santa Clara' considerada como padrão de sensibilidade a alta temperatura, de acordo com a literatura, e 'CL5915', padrão de tolerância, de acordo com Dane et al., (1991).

A semeadura foi realizada no município de Jaboticabal (SP), no mês de fevereiro de 1998, utilizando-se bandejas de poliestireno expandido, com 128 células, preenchidas com substrato para hortaliças (Plantmax). Com o surgimento da $3^{\text {a }}$ folha definitiva, as mudas foram transplantadas para vasos plásticos com $30 \mathrm{~cm}$ de diâmetro,com capacidade para $5 \mathrm{~kg}$, preenchidos com terra, adubo orgânico (3:1) e adubo químico (de acordo com as recomendações para a cultura) e levadas à casa-de-vegetação com controle de temperatura $\left(25^{\circ} \mathrm{C}\right)$ para avaliação das características associadas à germinação do pólen.

O experimento II foi conduzido em casade-vegetação, utilizando-se aquecedores para manter a temperatura acima de $20^{\circ} \mathrm{C}$ durante a noite. A fertilidade do solo foi corrigida com base na análise de solo e obedecendo às exigências da cultura. As mudas foram produzidas, como no experimento I, e transplantadas para o interior da casa-de- vegetação.
As adubações de cobertura e os defensivos agrícolas para controle de pragas e doenças foram utilizados de acordo com as recomendações para a cultura.

O ensaio para avaliar a germinação do pólen nas cultivares e na linhagem de tomateiro foi realizado no período de março a abril de 1998, utilizando-se a técnica de germinação in vitro.

A germinação de pólen in vitro constou no preparo de lâminas, com duas gotas de solução contendo $10 \%$ sacarose e $100 \mathrm{mgL}^{-1}$ de ácido bórico, na qual foram maceradas duas anteras, e avaliaram-se, sob microscópio óptico em aumento de 400 vezes, a germinação do pólen e o comprimento do tubo polínico.

Para essa técnica foram coletadas flores de cada genótipo e as lâminas foram preparadas logo após a coleta a $25^{\circ} \mathrm{C}$ (temperatura da casa-devegetação) e também após a exposição das flores a $30^{\circ} \mathrm{C}, 35^{\circ} \mathrm{C}, 40^{\circ} \mathrm{C}$ e $45^{\circ} \mathrm{C}$, por $1,2,3$ e 4 horas em câmara de germinação.

O meio de cultura utilizado foi preparado após a determinação das melhores concentrações de sacarose e ácido bórico, para se obter a maior porcentagem de germinação para os genótipos utilizados, baseando-se nas concentrações utilizadas por Trabelsi (1985).

O Experimento II foi conduzido para verificar se as respostas quanto à fixação de frutos coincidem com a resposta da viabilidade do pólen em altas temperaturas, permitindo uma diferenciação mais rápida entre os genótipos. Realizaram-se colheitas semanais, no primeiro mês de frutificação e, posteriormente, as colheitas foram realizadas quinzenalmente, avaliando-se o número de flores/rácimo e número de frutos fixados/rácimo, (frutos com diâmetro maior que $4 \mathrm{~mm}$ ).

O delineamento estatístico utilizado no Experimento I foi o inteiramente casualizado, com vinte e quatro tratamentos e três repetições. Os tratamentos nas diferentes temperaturas constaram de Genótipos (G) e Tempos de exposição $(\mathrm{H})$. Para cada temperatura avaliada entre $30^{\circ} \mathrm{C}$ e $45^{\circ} \mathrm{C}$, utilizou-se o delineamento inteiramente casualizado, arranjado de acordo com o esquema fatorial $6(\mathrm{G}) \times 4(\mathrm{H})+1$ testemunha $\left(25^{\circ} \mathrm{C}\right)$. No Experimento II foi utilizado o delineamento em 
blocos ao acaso, com seis genótipos e cinco repetições.

\section{RESULTADOS E DISCUSSÃO}

\section{Testes in vitro}

A germinação do pólen é expressa como o percentual total de grãos de pólen germinados em uma amostra. Verifica-se pelo Quadro 1 que não houve diferença significativa entre os genótipos utilizados, a $25^{\circ} \mathrm{C}$, quanto à porcentagem de germinação de pólen. Isso se deve ao fato de ser essa a condição ideal, uma vez que, segundo Aung (1978), para a germinação do pólen e crescimento do tubo polínico é necessário que a temperatura esteja entre 22 e $27^{\circ} \mathrm{C}$. Ao se observar o comportamento dos genótipos quando submetidos a $30^{\circ} \mathrm{C}$ por 1 hora, também não se verificaram diferenças significativas. Por outro lado, aumentando-se o tempo de exposição, as diferenças são visíveis para alguns genótipos, como, por exemplo, as cultivares TSW-10, Santa Clara e IPA-5 (2 horas) ou Príncipe Gigante (4 horas), que apresentaram menor porcentagem de germinação de pólen. Quanto aos tempos de exposição, verifica-se que 'Carmen', CL5915, 'IPA5' e 'TSW-10' não diferiram significativamente entre si em qualquer um dos tempos de exposição, fato esse que pode ser explicado pela superioridade desses genótipos em suportar as temperaturas excedentes à considerada favorável para a germinação do pólen.

No entanto, à medida que se aumenta a temperatura de exposição dos genótipos de $30^{\circ} \mathrm{C}$ para $35^{\circ} \mathrm{C}$, a porcentagem de germinação, que diminui em torno de $30 \%$, quando comparada à temperatura de $25^{\circ} \mathrm{C}$; sendo que com 3 e 4 horas de exposição, a maior porcentagem média de germinação foi verificada em 'Carmen' e CL5915, não diferindo de 'Santa Clara', com 4 horas de exposição. Esse fato evidencia o que foi relatado por Weaver (1989), segundo o qual a viabilidade do pólen decresce quando a temperatura do ar excede a $32^{\circ} \mathrm{C}$.

Por outro lado, quanto aos tempos de exposição, a porcentagem de germinação de pólen não foi afetada quando os genótipos CL5915, IPA5 e TSW-10 foram expostos a qualquer um dos tempos de exposição a $35^{\circ} \mathrm{C}$.

Observa-se uma significante diferença entre os genótipos a $40^{\circ} \mathrm{C}$, quanto à porcentagem de germinação de pólen. As cultivares Carmen e IPA-5 apresentaram a maior porcentagem média de germinação de pólen quando expostas a $40^{\circ} \mathrm{C}$ por 1 hora, enquanto que a menor porcentagem média foi verificada em 'TSW-10' nos tempos de exposição de 2, 3 e 4 horas. Weaver (1989) também verificou que a germinação de pólen e o comprimento do tubo polínico variaram entre as fontes de tomateiros expostos a $40^{\circ} \mathrm{C}$ por 60 minutos. Com relação ao tempo de exposição, verifica-se a superioridade da linhagem CL5915, que apresentou maior porcentagem de germinação, mesmo com 4 horas de exposição a $40^{\circ} \mathrm{C}$.

No entanto, quando foram submetidos a $45^{\circ} \mathrm{C}$, pôde-se estabelecer o nível de tolerância ao calor dos genótipos, identificando aqueles que apresentaram a maior porcentagem de germinação do pólen no maior tempo de exposição. Entre os genótipos estudados, verificou-se que com 4 horas de exposição a $45^{\circ} \mathrm{C}$, a maior porcentagem média foi observada em CL5915. Por outro lado, tornouse evidente a sensibilidade de 'Santa Clara' quando exposta a essa temperatura. Entretanto, a $45^{\circ} \mathrm{C}$, a porcentagem de germinação de pólen foi quase que totalmente inibida na maioria dos genótipos estudados.

O crescimento do tubo polínico foi fator considerado por Smith (1935) c omo comprometedor do pegamento de frutos. Sob temperatura ótima de germinação, ou seja, $21^{\circ} \mathrm{C}$, foram observados tubos polínicos de $2 \mathrm{~mm}$. Para os genótipos estudados, pode-se observar no Quadro 2 que, quando foram submetidos a $25^{\circ} \mathrm{C}$ não houve diferença significativa entre os genótipos, o que foi diferente do observado quando os genótipos foram expostos a $30^{\circ} \mathrm{C}$. Porém, quanto aos diferentes tempos de exposição, observa-se a superioridade de 'CL5915' e 'Santa Clara', que não diferiram significativamente de 'Carmen', 'IPA-5' e 'Príncipe Gigante', quanto ao comprimento do tubo polínico. Reghin (1996) cita que temperaturas acima de $27^{\circ} \mathrm{C}$ retardam tanto a germinação do pólen como o crescimento do tubo polínico. 
QUADRO 1 - Médias da porcentagem de germinação de pólen, em lâminas, dos genótipos de tomateiro, quando o pólen foi exposto a $25,30,35,40$ e $45^{\circ} \mathrm{C}$, por 1, 2, 3 e 4 horas.

\begin{tabular}{|c|c|c|c|c|c|c|}
\hline \multirow[b]{2}{*}{ Temperatura } & \multicolumn{6}{|c|}{ Genótipos } \\
\hline & 'Carmen' & CL5915 & 'IPA-5' & $\begin{array}{l}\text { 'Príncipe } \\
\text { Gigante' }\end{array}$ & 'Santa Clara' & 'TSW-10' \\
\hline $25^{\circ} \mathrm{C}(\mathrm{T})$ & $84,36 \mathrm{~A}$ & $85,68 \mathrm{~A}$ & $83,68 \mathrm{~A}$ & $81,04 \mathrm{~A}$ & $82,76 \mathrm{~A}$ & $83,10 \mathrm{~A}$ \\
\hline DMS (5\%) & \multicolumn{2}{|c|}{5,1168} & & & & \\
\hline $30^{\circ} \mathrm{C} / 1 \mathrm{~h}$ & $71,54 \mathrm{~A}^{1} \mathrm{a}^{2}$ & $71,65 \mathrm{Aa}$ & $72,29 \mathrm{Aa}$ & $70,35 \mathrm{Aab}$ & $70,29 \mathrm{Aab}$ & $70,47 \mathrm{Aa}$ \\
\hline $30^{\circ} \mathrm{C} / 2 \mathrm{~h}$ & $72,90 \mathrm{Aa}$ & $71,72 \mathrm{ABa}$ & $70,61 \mathrm{ABCa}$ & $71,89 \mathrm{ABa}$ & $69,95 \mathrm{BCab}$ & $68,56 \mathrm{Ca}$ \\
\hline $30^{\circ} \mathrm{C} / 3 \mathrm{~h}$ & $72,23 \mathrm{Aa}$ & $71,10 \mathrm{ABa}$ & $71,05 \mathrm{ABa}$ & $70,34 \mathrm{ABab}$ & $69,33 \mathrm{Bb}$ & $68,67 \mathrm{Ba}$ \\
\hline $30^{\circ} \mathrm{C} / 4 \mathrm{~h}$ & $71,06 \mathrm{ABa}$ & $72,65 \mathrm{Aa}$ & $70,24 \mathrm{ABCa}$ & $69,08 \mathrm{BCb}$ & $71,56 \mathrm{Aa}$ & $68,42 \mathrm{Ca}$ \\
\hline DMS (5\%) & \multicolumn{2}{|c|}{2,4720} & \multicolumn{4}{|c|}{ Genótipos dentro do tempo de exposição } \\
\hline DMS (5\%) & \multicolumn{2}{|c|}{2,2186} & \multicolumn{4}{|c|}{ Tempo de exposição dentro de genótipo } \\
\hline $35^{\circ} \mathrm{C} / 1 \mathrm{~h}$ & $61,16 \mathrm{Aa}$ & $57,42 \mathrm{BCa}$ & $57,83 \mathrm{Ba}$ & $56,30 \mathrm{BCab}$ & $55,96 \mathrm{BCb}$ & $54,58 \mathrm{Ca}$ \\
\hline $35^{\circ} \mathrm{C} / 2 \mathrm{~h}$ & $61,75 \mathrm{Aa}$ & $57,50 \mathrm{BCa}$ & $56,83 \mathrm{BCa}$ & $59,20 \mathrm{ABa}$ & $54,74 \mathrm{CDb}$ & $52,31 \mathrm{Da}$ \\
\hline $35^{\circ} \mathrm{C} / 3 \mathrm{~h}$ & $59,68 \mathrm{Aab}$ & $57,42 \mathrm{ABa}$ & $55,76 \mathrm{Ba}$ & $55,98 \mathrm{Bb}$ & $54,74 \mathrm{BCb}$ & $51,79 \mathrm{Ca}$ \\
\hline $35^{\circ} \mathrm{C} / 4 \mathrm{~h}$ & $57,76 \mathrm{ABb}$ & $58,90 \mathrm{Aa}$ & $54,97 \mathrm{BCa}$ & $54,13 \mathrm{Cb}$ & $58,13 \mathrm{Aa}$ & $51,95 \mathrm{Ca}$ \\
\hline DMS (5\%) & \multicolumn{2}{|c|}{3,2466} & \multicolumn{4}{|c|}{ Genótipos dentro do tempo de exposição } \\
\hline DMS (5\%) & \multicolumn{2}{|c|}{2,9138} & \multicolumn{4}{|c|}{ Tempo de exposição dentro de genótipo } \\
\hline $40^{\circ} \mathrm{C} / 1 \mathrm{~h}$ & $51,81 \mathrm{Ab}$ & $46,23 \mathrm{Bc}$ & $51,22 \mathrm{Aa}$ & $47,04 \mathrm{Bb}$ & $41,99 \mathrm{Cb}$ & $39,72 \mathrm{Ca}$ \\
\hline $40^{\circ} \mathrm{C} / 2 \mathrm{~h}$ & $59,17 \mathrm{Aa}$ & $46,21 \mathrm{Cc}$ & $47,11 \mathrm{Cb}$ & $51,38 \mathrm{Ba}$ & $44,37 \mathrm{Cb}$ & $38,29 \mathrm{Dab}$ \\
\hline $40^{\circ} \mathrm{C} / 3 \mathrm{~h}$ & $53,64 \mathrm{Ab}$ & $50,81 \mathrm{ABb}$ & $45,82 \mathrm{Db}$ & $47,00 \mathrm{CDb}$ & $49,67 \mathrm{BCa}$ & $35,30 \mathrm{~Eb}$ \\
\hline $40^{\circ} \mathrm{C} / 4 \mathrm{~h}$ & $51,05 \mathrm{Bb}$ & $57,84 \mathrm{Aa}$ & $44,94 \mathrm{Cb}$ & $42,25 \mathrm{Cc}$ & $42,65 \mathrm{Cb}$ & $36,83 \mathrm{Dab}$ \\
\hline DMS (5\%) & \multicolumn{2}{|c|}{3,5669} & \multicolumn{4}{|c|}{ Genótipos dentro do tempo de exposição } \\
\hline DMS (5\%) & \multicolumn{2}{|c|}{3,2013} & \multicolumn{4}{|c|}{ Tempo de exposição dentro de genótipo } \\
\hline $45^{\circ} \mathrm{C} / 1 \mathrm{~h}$ & $25,59 \mathrm{Abc}$ & $22,26 \mathrm{ABb}$ & $20,13 \mathrm{Ba}$ & $17,84 \mathrm{Bb}$ & $12,69 \mathrm{Cb}$ & $8,48 \mathrm{Cab}$ \\
\hline $45^{\circ} \mathrm{C} / 2 \mathrm{~h}$ & $36,39 \mathrm{Aa}$ & $19,19 \mathrm{Cb}$ & $16,16 \mathrm{Cdab}$ & $27,02 \mathrm{Ba}$ & $11,57 \mathrm{DEb}$ & $10,24 \mathrm{Ea}$ \\
\hline $45^{\circ} \mathrm{C} / 3 \mathrm{~h}$ & $27,56 \mathrm{Ab}$ & $22,42 \mathrm{Bb}$ & $14,74 \mathrm{Cb}$ & $17,87 \mathrm{BCb}$ & $9,69 \mathrm{Db}$ & $8,13 \mathrm{Dab}$ \\
\hline $45^{\circ} \mathrm{C} / 4 \mathrm{~h}$ & $22,34 \mathrm{Bc}$ & $32,97 \mathrm{Aa}$ & $13,57 \mathrm{Cb}$ & $14,98 \mathrm{Bb}$ & $20,94 \mathrm{Ba}$ & $5,18 \mathrm{Db}$ \\
\hline DMS (5\%) & \multicolumn{2}{|c|}{5,0201} & \multicolumn{4}{|c|}{ Genótipos dentro do tempo de exposição } \\
\hline DMS (5\%) & \multicolumn{2}{|c|}{4,5055} & \multicolumn{4}{|c|}{ Tempo de exposição dentro de genótipo } \\
\hline
\end{tabular}


À medida que aumentou a temperatura de exposição, os genótipos submetidos a $35^{\circ} \mathrm{C}$ por 1 hora, como 'IPA-5', 'Carmen' e CL5915, apresentaram o maior comprimento médio do tubo polínico, não diferindo significativamente entre si, enquanto que quando submetidos a 4 horas de exposição, apenas CL5915 apresentou superioridade no comprimento médio do tubo polínico. Por outro lado, quanto aos tempos de exposição, 'TSW-10' não mostrou diferenças significativas quando submetidas a qualquer um dos tempos de exposição a $35^{\circ} \mathrm{C}$, enfocando que essa temperatura de exposição é limitante ao crescimento do tubo polínico em qualquer um dos tempos a que a cultivar foi submetida.

No entanto, a exposição a $40^{\circ} \mathrm{C}$ favoreceu ainda mais a redução no comprimento médio do tubo polínico, o que foi verificado em todos os genótipos; entre eles, podemos enfocar a inferioridade de ' TSW-10', ' Príncipe Gigante' e ' IPA-5', quanto ao comprimento médio do tubo polínico. Quanto aos tempos de exposição, permaneceu o já observado a $35^{\circ} \mathrm{C}$. A cultivar TSW-10 não diferiu significativamente quanto ao comprimento médio do tubo polínico em qualquer um dos tempos de exposição.

A exposição das cultivares a $45^{\circ} \mathrm{C}$ favoreceu a seleção efetiva dos genótipos que, mesmo expostos ao maior tempo (4 horas), sobressaíram-se, apresentando o maior comprimento médio do tubo polínico; entre eles, estão CL5915 e ' Carmen', que não diferiram significativamente entre si. Quanto aos tempos de exposição, 'IPA-5', 'Príncipe Gigante', 'Santa Clara' e 'TSW-10' não diferiram significativamente entre si, quando expostas a qualquer um dos tempos de exposição, confirmando a influência da alta temperatura no desenvolvimento do tubo polínico.

A germinação de pólen e o comprimento do tubo polínico diminuíram significativamente com o aumento da temperatura, concordando com os resultados obtidos por Smith e Cochran (1935).

\section{Avaliações em casa-de-vegetação}

\section{Número de Flores/Rácimo}

Conforme pode ser observado, no Quadro 3, as cultivares Carmen e IPA-5 apresentam o menor número médio de flores/rácimo, quando cultivadas sob condições de alta temperatura em casa-de-vegetação, enquanto Santa Clara apresentou o maior número, entretanto, não diferindo significativamente de 'Príncipe Gigante' e CL5915. Porém, esse fato não é fundamental para se conseguir uma boa produção, porque, como relatam Hurd e Cooper, citados por Monselise (1986), o rendimento de tomate não é determinado pelo número de flores produzidas por planta, mas pela porcentagem de frutos fixados e pelo desenvolvimento dos frutos. Comparando-se o número de flores por rácimo com a porcentagem de germinação de pólen, verifica-se que 'Santa Clara' apresentou o maior número de flores; no entanto, essa cultivar se encontra entre as que apresentaram menor porcentagem de grãos de pólen que germinaram em condições de alta temperatura.

\section{Número de Frutos Fixados/Rácimo}

Sob condições de casa-de-vegetação, os genótipos de tomateiro diferiram amplamente na fixação de frutos.

De acordo com os resultados, mostrados também no Quadro 3, a linhagem CL5915 apresentou o maior número médio de frutos fixados/rácimo (3,70), seguida pela cultivar Carmen' (1,74). Abdul-Baki e Stommel (1995) observaram, em tomateiros expostos a altas temperaturas, que não ocorreu pegamento de fruto nos genótipos mais sensíveis ao calor. Os resultados observados evidenciam que os melhores valores de germinação e viabilidade de pólen para essa linhagem demonstraram sua maior tolerância ao estresse térmico. 
QUADRO 2 - Comprimento médio do tubo polínico, em lâminas, de genótipos de tomateiro expostos a 25, 30, 35, 40 e $45^{\circ} \mathrm{C}$, por $1,2,3$ e 4 horas.

\begin{tabular}{|c|c|c|c|c|c|c|}
\hline \multirow{2}{*}{ Temperatura } & \multicolumn{6}{|c|}{ Genótipos } \\
\hline & 'Carmen' & CL5915 & 'IPA-5' & $\begin{array}{l}\text { 'Príncipe } \\
\text { Gigante' }\end{array}$ & $\begin{array}{l}\text { 'Santa } \\
\text { Clara' }\end{array}$ & 'TSW-10' \\
\hline $25^{\circ} \mathrm{C}(\mathrm{T})$ & $1,38 \mathrm{~A}$ & $1,39 \mathrm{~A}$ & $1,39 \mathrm{~A}$ & $1,38 \mathrm{~A}$ & $1,38 \mathrm{~A}$ & $1,38 \mathrm{~A}$ \\
\hline DMS (5\%) & \multicolumn{2}{|c|}{$\mathbf{0 , 0 1 7 1}$} & & & & \\
\hline $30^{\circ} \mathrm{C} / 1 \mathrm{~h}$ & $1,37 \mathrm{AB}^{1} \mathrm{~b}^{2}$ & $1,38 \mathrm{Aa}$ & $1,38 \mathrm{Aa}$ & $1,36 \mathrm{Bb}$ & $1,37 \mathrm{ABab}$ & $1,37 \mathrm{ABa}$ \\
\hline $30^{\circ} \mathrm{C} / 2 \mathrm{~h}$ & $1,38 \mathrm{Aa}$ & $1,38 \mathrm{Aa}$ & $1,37 \mathrm{Bb}$ & $1,38 \mathrm{Aa}$ & $1,37 \mathrm{Bab}$ & $1,37 \mathrm{Ba}$ \\
\hline $30^{\circ} \mathrm{C} / 3 \mathrm{~h}$ & $1,38 \mathrm{Aa}$ & $1,37 \mathrm{ABb}$ & $1,38 \mathrm{Aa}$ & $1,38 \mathrm{Aa}$ & $1,36 \mathrm{Bb}$ & $1,37 \mathrm{ABa}$ \\
\hline $30^{\circ} \mathrm{C} / 4 \mathrm{~h}$ & $1,37 \mathrm{ABb}$ & $1,38 \mathrm{Aa}$ & $1,37 \mathrm{ABb}$ & $1,37 \mathrm{ABab}$ & $1,38 \mathrm{Aa}$ & $1,36 \mathrm{Bb}$ \\
\hline DMS (5\%) & \multicolumn{2}{|c|}{0,0107} & \multicolumn{4}{|c|}{ Genótipos dentro do tempo de exposição. } \\
\hline DMS (5\%) & \multicolumn{2}{|c|}{0,0096} & \multicolumn{4}{|c|}{ Tempo de exposição dentro de genótipo. } \\
\hline $35^{\circ} \mathrm{C} / 1 \mathrm{~h}$ & $1,15 \mathrm{ABbc}$ & $1,14 \mathrm{ABCb}$ & $1,17 \mathrm{Aa}$ & $1,13 \mathrm{BCD}$ & $1,12 \mathrm{Cdab}$ & $1,11 \mathrm{Da}$ \\
\hline $35^{\circ} \mathrm{C} / 2 \mathrm{~h}$ & 1,19 Aab & $1,15 \mathrm{Bb}$ & $1,14 \mathrm{Bab}$ & $1,15 \mathrm{Ba}$ & $1,10 \mathrm{Cb}$ & $1,11 \mathrm{Ca}$ \\
\hline $35^{\circ} \mathrm{C} / 3 \mathrm{~h}$ & $1,18 \mathrm{Aab}$ & $1,15 \mathrm{Bb}$ & $1,14 \mathrm{Bb}$ & $1,14 \mathrm{Ba}$ & $1,10 \mathrm{Cb}$ & $1,09 \mathrm{Ca}$ \\
\hline $35^{\circ} \mathrm{C} / 4 \mathrm{~h}$ & $1,15 \mathrm{Bc}$ & $1,19 \mathrm{Aa}$ & $1,15 \mathrm{Bb}$ & $1,14 \mathrm{Ba}$ & $1,14 \mathrm{Ba}$ & $1,10 \mathrm{Ca}$ \\
\hline DMS (5\%) & \multicolumn{2}{|c|}{0,0256} & \multicolumn{4}{|c|}{ Genótipos dentro do tempo de exposição. } \\
\hline DMS (5\%) & \multicolumn{2}{|c|}{0,0230} & \multicolumn{4}{|c|}{ Tempo de exposição dentro de genótipo. } \\
\hline $40^{\circ} \mathrm{C} / 1 \mathrm{~h}$ & $1,06 \mathrm{ABb}$ & $1,05 \mathrm{ABb}$ & $1,07 \mathrm{Aa}$ & $1,06 \mathrm{ABab}$ & $1,03 \mathrm{Bb}$ & $1,04 \mathrm{ABa}$ \\
\hline $40^{\circ} \mathrm{C} / 2 \mathrm{~h}$ & $1,13 \mathrm{Aa}$ & $1,05 \mathrm{BCb}$ & $1,06 \mathrm{BCab}$ & $1,08 \mathrm{Ba}$ & $1,03 \mathrm{Cb}$ & $1,04 \mathrm{Ca}$ \\
\hline $40^{\circ} \mathrm{C} / 3 \mathrm{~h}$ & $1,09 \mathrm{Ab}$ & $1,06 \mathrm{ABb}$ & $1,05 \mathrm{Bab}$ & $1,06 \mathrm{ABab}$ & $1,05 \mathrm{Bab}$ & $1,04 \mathrm{Ba}$ \\
\hline $40^{\circ} \mathrm{C} / 4 \mathrm{~h}$ & $1,07 \mathrm{ABb}$ & $1,10 \mathrm{Aa}$ & $1,04 \mathrm{BCb}$ & $1,05 \mathrm{BCb}$ & $1,07 \mathrm{ABa}$ & $1,03 \mathrm{Ca}$ \\
\hline DMS (5\%) & \multicolumn{2}{|c|}{$\mathbf{0 , 0 3 7 7}$} & \multicolumn{4}{|c|}{ Genótipos dentro do tempo de exposição. } \\
\hline DMS (5\%) & \multicolumn{2}{|c|}{$\mathbf{0 , 0 3 3 8}$} & \multicolumn{4}{|c|}{ Tempo de exposição dentro de genótipo. } \\
\hline $45^{\circ} \mathrm{C} / 1 \mathrm{~h}$ & $1,01 \mathrm{Ab}$ & $1,00 \mathrm{Ab}$ & $1,00 \mathrm{Aa}$ & $1,01 \mathrm{Aa}$ & $1,01 \mathrm{Aa}$ & $1,00 \mathrm{Aa}$ \\
\hline $45^{\circ} \mathrm{C} / 2 \mathrm{~h}$ & $1,03 \mathrm{Aa}$ & $1,00 \mathrm{Bb}$ & $1,00 \mathrm{Ba}$ & $1,00 \mathrm{Ba}$ & $1,00 \mathrm{Ba}$ & $1,00 \mathrm{Ba}$ \\
\hline $45^{\circ} \mathrm{C} / 3 \mathrm{~h}$ & $1,01 \mathrm{Ab}$ & $1,00 \mathrm{Ab}$ & $1,00 \mathrm{Aa}$ & $1,00 \mathrm{Aa}$ & $1,00 \mathrm{Aa}$ & $1,00 \mathrm{Aa}$ \\
\hline $45^{\circ} \mathrm{C} / 4 \mathrm{~h}$ & $1,01 \mathrm{ABb}$ & $1,02 \mathrm{Aa}$ & $1,00 \mathrm{Ba}$ & $1,00 \mathrm{Ba}$ & $1,00 \mathrm{Ba}$ & $1,00 \mathrm{Ba}$ \\
\hline DMS (5\%) & \multicolumn{2}{|c|}{0,0119} & \multicolumn{4}{|c|}{ Genótipos dentro do tempo de exposição. } \\
\hline DMS (5\%) & \multicolumn{2}{|c|}{0,0107} & \multicolumn{4}{|c|}{ Tempo de exposicão dentro de genótipo. } \\
\hline
\end{tabular}

R. Bras. Fisiol. Veg., 12(2):156-165, 2000. 
QUADRO 3 - Médias do $\mathrm{n}^{\circ}$ de flores/rácimo e $\mathrm{n}^{\circ}$ de frutos fixados/rácimo de plantas de genótipos de tomateiro cultivados em casa-de-vegetação sob condições de altas temperaturas.

\begin{tabular}{lcc}
\hline \multicolumn{1}{c}{ Genótipos } & $\mathbf{N}^{\mathbf{0}}$ de flores/ rácimo & $\mathbf{N}^{\mathbf{0}}$ de frutos fixados/ Rácimo \\
\hline 'Carmen' & $6,60 \mathrm{C}$ & $1,74 \mathrm{~B}$ \\
CL 5915 & $9,25 \mathrm{~A}$ & $3,70 \mathrm{~A}$ \\
'IPA-5' & $6,61 \mathrm{C}$ & $0,42 \mathrm{C}$ \\
'Príncipe Gigante' & $9,32 \mathrm{~A}$ & $0,31 \mathrm{C}$ \\
'Santa Clara' & $9,98 \mathrm{~A}$ & $0,25 \mathrm{C}$ \\
'TSW-10' & $8,40 \mathrm{~B}$ & $0,68 \mathrm{C}$ \\
\hline D.M.S. (5\%) & $\mathbf{0 , 8 0 6 5}$ & $\mathbf{0 , 8 0 1 9}$ \\
\hline
\end{tabular}

${ }^{1}$ Médias com a mesma letra maiúscula nas colunas não diferem entre si ao nível de $5 \%$ de probabilidade pelo teste de Tukey.

Analisando o número de flores/rácimo e o número de frutos fixados, verifica-se que a porcentagem média de fixação de frutos variou de $2,50 \%$ em 'Santa Clara' a 40\% em CL5915, o que evidencia a melhor performance dessa linhagem, quando cultivada sob condições de alta temperatura. A cultivar Santa Clara, apesar de apresentar o maior número de flores por rácimo, não apresentou a maior porcentagem média de fixação de frutos. Segundo Peterson \& Taber (1987), citados por Martins (1992), em altas temperaturas do ar ocorre menor formação de frutos por cachos, comparada às temperaturas normais, $22^{\circ} \mathrm{C}$ durante o dia. Esse fato é explicado pela protusão do estigma, resultando em insucesso na polinização, e pelo lento crescimento do tubo polínico, no caso de haver polinização, resultando em menor pegamento de fruto, concordando com Lohar e Peat (1998), que verificaram a protusão do estigma em altas temperaturas $\left(28 / 23^{\circ} \mathrm{C}\right) \mathrm{em}$ genótipos de tomateiro, resultando em menor fixação de frutos. A linhagem CL5915 foi o genótipo significativamente superior quanto à capacidade em fixar frutos sob elevadas temperaturas.

\section{CONCLUSÕES}

Pela observação dos resultados obtidos por meio da técnica de germinação do pólen in vitro e para a porcentagem de fixação de frutos no cultivo in vivo em casa-de-vegetação, pode se concluir que:

De maneira geral, a técnica de germinação in vitro possibilita a identificação dos genótipos tolerantes e dos sensíveis à alta temperatura, confirmando o corrigido em casa-devegetação, podendo, dessa forma, ser utilizada como indicativo de tolerância à alta temperatura em genótipos de tomateiro. Por outro lado, diferenças quanto aos resultados entre as técnicas in vitro $e$ in vivo foram verificadas. Esse fato pode ser devido às condições de temperatura na casa-devegetação não serem exatamente as mesmas a que os genótipos foram submetidos no laboratório, como, por exemplo, permanecer um longo período na mesma temperatura, sem oscilações.

\section{AGRADECIMENTO}

\section{À FAPESP.}




\section{REFERÊNCIAS}

ABDALLA, A.A., VERKERK, K. Growth flowering and fruit set of the tomato at high temperature. Neth. J. Agric. Sci., v. 16, p.716, 1968.

ABDUL-BAKI, A.; STOMMEL, J.R. Pollen viability and fruit set of tomato genotypes under optimum and high-temperature regimes. HortScience, v.30, n.1, p.115-17, 1995.

AGRIANUAL 2000. São Paulo: FNP, 2000. p.515-26.

ANDERLINI, R. A cultura do tomate. Lisboa:Litexa, 1982. 164p.

AUNG, L.H. Temperature regulation of growth and development of tomato during ontogeny. In: INTERNATIONAL SYMPOSIUM ON TROPICAL TOMATO, 1, Shanhura, Taiwan, p.23-7, 1978. Proceedings...

BRAZ, L.T. Caracterização de genótipos (Lycopersicon esculentum Mill.) em cultivo no Brasil quanto a tolerância à alta temperatura. Jaboticabal, 1995. p55. (Relatório apresentado à Faculdade de Ciências Agrárias e Veterinárias, UNESP, Campus de Jaboticabal, para cumprir as exigências do plano global das atividades referentes ao triênio de 92/95).

DANE, A.; HUNTER, G., CHAMBLISS, O. L. Fruit set, pollen fertility, and combining ability of selected tomato genotypes under hightemperature field conditions. J. Am. Soc. Hortic., v.116, n.5, p.906-10, 1991.

IWAHORI, S. High temperature injury in tomato. J. of the Japanese Soc. for Hortic. Sci., v.34, p.33-41, 1965.

LOHAR,D.P.; PEAT, W. E. Floral characteristics of heat-tolerant and heat-sensitive tomato (Lycopersicon esculentum Mill.) cultivars at high temperature. Sci. Hortic., v.73, p.53-60, 1998.
MARTINS, G. Uso de casa de vegetação com cobertura plástica na tomaticultura de verão. Jaboticabal, 1992. 65p. Tese (Doutorado em Agronomia)-Faculdade de Ciências Agrárias e Veterinárias, Universidade Estadual Paulista.

MONSELISE, S. P. CRC Handdbook of fruit set and development. Florida: CRC Press, p.46080, 1986.

REGHIN, M. Y. Fisiologia do desenvolvimento das hortaliças em ambiente protegido. Botucatu: Departamento de Horticultura, Faculdade de Ciências Agronômicas, Universidade Estadual Paulista, 1996, 12p. (mimiografado)

SMITH, O.; COCHRAN, H.L. Temperature and pollen germination in the tomato. Cornell: Agric. Exp. Stat., 1935. v.175. 11p.

SMITH, O. Pollination and life-history studies of the tomato (Lycopersicon esculentum Mill.). Cornell: Univ. Agric. Exp. Stat., 1935. p.3-16 (Mem., 184).

STEVENS, M.A., RUDICH, J. Genetic potencial for overcoming physiological limitations on adaptability, yield, and quality in the tomato. HortScience, Alexandria, v.13, p.673-78, 1978.

SUGIYAMA, T.; IWAHORI, S.; TAKAHASHI, $\mathrm{K}$. Effect of high temperature on fruit setting of tomato under cover. Acta Hortic., v.4, p.63-9, 1966.

TIGCHELAAR, E.C. Tomato breeding. In: BASSET, M.J. Breeding vegetable crops. Westport: AVI Publishing Company, 1986. p.135-66.

TRABELSI, M. A reliable method for testing fruit setting ability in tomato using "in vitro" pollen germination. Meded. Fac. Landbouwwet. Rijksuniv. Gent, Ghent, v.50 
n.4, p.1343-56,1985. In: Hortic. Abstr., WEAVER, M. L. Screening tomato for highFarnham Royal, v.56, n.6, p.466, $1986 . \quad$ temperature tolerance through pollen viability (Abstrat, 4330). testes. HortScience, v. 24, n.3, p.493-5, 1989. 